\title{
Focused Assessment with Sonography in Trauma (FAST) Training for First-Year Resident Physicians at a University Hospital in Japan: A Longitudinal, Observational Study
}

\section{Koshi Ota ( $\square$ emm006@osaka-med.ac.jp)}

Osaka Medical College: Osaka Ika Daigaku https://orcid.org/0000-0002-1461-7031

\section{Koji Oba}

The University of Tokyo: Tokyo Daigaku

\section{Yuri lto}

Osaka Medical College: Osaka Ika Daigaku

\section{Kanna Ota}

Osaka Medical College: Osaka Ika Daigaku

\section{Akira Takasu}

Osaka Medical College: Osaka Ika Daigaku

\section{Research article}

Keywords: Emergency medicine, Focused Assessment with Sonography for Trauma (FAST), Postgraduate year 1, Ultrasound

Posted Date: October 20th, 2020

DOI: https://doi.org/10.21203/rs.3.rs-92189/v1

License: (c) (i) This work is licensed under a Creative Commons Attribution 4.0 International License.

Read Full License

Version of Record: A version of this preprint was published at SAGE Open Medicine on January 1st, 2021. See the published version at https://doi.org/10.1177/20503121211044367. 


\section{Abstract}

Background: Ultrasound training is an essential part of residency programs during emergency medicine (EM) rotations for first-year trainees (postgraduate year 1; PGY1). The Focused Assessment with Sonography for Trauma (FAST) examination to assess for internal bleeding in trauma patients is one of the essential scans, which PGY1 residents must learn during the EM rotation.

Method: A prospective, longitudinal, observational study of PGY1 residents during an EM rotation (two months) conducted from April 1, 2019 to March 31, 2020 was performed. The primary outcome was the mean difference between the hands-on FAST examination scores of the first week of the EM rotation and the same hands-on FAST examination scores of the last week of the EM rotation. All PGY1 residents could access the ultrasound machine freely for examining other PGY1 residents and could use it for real patients under supervision of EM physicians.

Result: A total of 34 PGY1 residents (male 29, female 5) were recruited and completed the hands-on FAST test in both the first week and the last week of the rotation. The mean test score for the PGY1 residents in the first week was 7.53 (standard deviation [SD] 2.12). The mean test score in the last week was 15.88 (SD 2.46). The primary outcome of this study was the score difference between the first and last weeks (mean 8.35, 95\% confidence interval 7.36 - 9.35, $\mathrm{P}<0.0001$, paired $t$-test).

Conclusion: Hands-on practical FAST training for PGY1 residents during EM rotations significantly improved their FAST test scores.

\section{Introduction}

Ultrasound training is an essential part of residency programs during emergency medicine (EM) rotations for first-year trainees (postgraduate year 1; PGY1). ${ }^{1}$ The Focused Assessment with Sonography for Trauma (FAST) examination to assess for internal bleeding in trauma patients is one of the essential scans, which PGY1 residents must learn during their EM rotation due to its time-efficiency and noninvasiveness. ${ }^{23}$

Although FAST training should be done during medical school, ${ }^{4}$ the majority of PGY1 residents have not received formal education in ultrasound examinations.

In Japan, all doctors have been mandated to have 2 years of official postgraduate clinical training since 2004. PGY1 and PGY2 resident have to rotate in major specialties, including $E M$, internal medicine, pediatrics, surgery, and community-based practice. ${ }^{5}$ The duration of the EM rotation is at the discretion of each hospital. The EM rotation of our institution is 2 months and is mandatory for PGY1 residents.

There are many factors that interfere with the opportunity to gain experience with FAST training in the real-time ED setting, including seasonal volume changes of patients, the fast pace of care, and the number of PGY1 residents during the rotation. Given these barriers, it is not common for PGY1 residents 
to complete an EM rotation with adequate experience with real patients; thus, we offered PGY1 residents the opportunity to freely use the ultrasound machine during their rotation for practice by themselves. The EM rotation provides an excellent opportunity for FAST training. ${ }^{67}$ The purpose of this study was to assess the effectiveness of live patient-based FAST training for PGY1 residents within an established EM rotation.

\section{Materials And Methods}

A prospective, longitudinal, observational study of PGY1 residents during EM rotations conducted from April 1, 2019 to March 31, 2020 (2019-2020 fiscal year) was performed. Each rotation lasted for 2 months. The first hands-on FAST examination was implemented without lectures, so that PGY1 residents would perform the FAST using the skill and knowledge that they had learned in medical school. All participating PGY1 residents took a standardized list of key assessment points that was provided verbally and were instructed interactively after the first test to ensure consistency in the teaching points in the first week of the rotation. Although simulation-based training showed better performance than normal training without simulation-based training in diagnostic ultrasound scanning on real patients, ${ }^{8}$ PGY1 residents used the ultrasound machine for real patients only.

The ultrasound machine used in the emergency room was a GE LOGIQ E9 XDclear 2.0 ultrasound machine (GE Healthcare, Wauwatosa, WI, USA), and the PGY1 residents could use the machine for real patients under the supervision of attending EM physicians or for other PGY1 residents freely without supervision. The instructor did all scoring in real time. This study was conducted at an academic university hospital and was approved by the Institutional Review Board (2767), with the written, informed consent of all participants.

\section{Participants}

The participants were first-year rotating residents (PGY1) $(n=34)$ in the EM rotation at an academic university hospital during the 2019-2020 fiscal year. Table 1 shows information regarding the identified specialty of interest at the time of the rotation of the PGY1 residents. They were divided three categories, internal medicine group, surgery group, and others group. The internal medicine group included cardiology, gastroenterology, respiratory medicine, nephrology, neurology, hematology, and rheumatology. The surgery group included cardiovascular surgery, thoracic surgery, gastroenterological surgery, breast and endocrine surgery, orthopedic surgery, neurosurgery, obstetrics and gynecology, and emergency medicine. The others group included pediatrics, anesthesiology, ophthalmology, otorhinolaryngology, dermatology, plastic surgery, radiology, and psychiatry. 
Table 1

Demographic and characteristics of the postgraduate year 1 residents.

Resident's identified specialty of interest at the time of

rotation

\begin{tabular}{|c|c|c|c|c|}
\hline & Internal medicine & Surgery & Others & $\begin{array}{l}\text { Fisher exact } \\
\text { test }\end{array}$ \\
\hline Sex & & & & 0.395 \\
\hline female & 1 & 0 & 4 & \\
\hline male & 7 & 9 & 13 & \\
\hline Group & & & & 0.169 \\
\hline April-May & 0 & 3 & 4 & \\
\hline June-July & 1 & 1 & 4 & \\
\hline $\begin{array}{l}\text { August- } \\
\text { September }\end{array}$ & 1 & 3 & 1 & \\
\hline $\begin{array}{l}\text { October- } \\
\text { November }\end{array}$ & 1 & 1 & 3 & \\
\hline $\begin{array}{l}\text { December- } \\
\text { January }\end{array}$ & 1 & 0 & 4 & \\
\hline February-March & 4 & 1 & 1 & \\
\hline Evaluation & & & & 0.799 \\
\hline 1 & 0 & 0 & 1 & \\
\hline 2 & 0 & 1 & 1 & \\
\hline 3 & 3 & 4 & 9 & \\
\hline 4 & 4 & 3 & 6 & \\
\hline
\end{tabular}

*Internal medicine includes cardiology, gastroenterology, respiratory medicine, nephrology, neurology, hematology and rheumatology.

** Surgery includes cardiovascular surgery, thoracic surgery, gastroenterological surgery, breast and endocrine surgery, orthopedic surgery, neurosurgery, obstetrics and gynecology, and emergency medicine.

*** Others include pediatrics, anesthesiology, ophthalmology, otorhinolaryngology, dermatology, plastic surgery, radiology and psychiatry.

${ }^{\dagger}$ Evaluation category means the average score by three third year EM residents for three-item survey regarding their clinical attitude, knowledge and skill for EM, and positive participation to EM practice on a scale of one to five with 5 being best. 1 means average score was greater than or equal to 1 and less than 2. 2 means average score was greater than or equal to 2 and less than 3 . 3 means average score was greater than or equal to 3 and less than 5.4 means average score was greater than or equal to 4 and less than 5.5 means average score was 5 . 


\begin{tabular}{|c|c|c|}
\hline \multicolumn{3}{|c|}{$\begin{array}{l}\text { Resident's identified specialty of interest at the time of } \\
\text { rotation }\end{array}$} \\
\hline 5 & 1 & 0 \\
\hline \multicolumn{3}{|c|}{$\begin{array}{l}\text { *Internal medicine includes cardiology, gastroenterology, respiratory medicine, nephrology, neurology, } \\
\text { hematology and rheumatology. }\end{array}$} \\
\hline \multicolumn{3}{|c|}{$\begin{array}{l}\text { ** Surgery includes cardiovascular surgery, thoracic surgery, gastroenterological surgery, breast and } \\
\text { endocrine surgery, orthopedic surgery, neurosurgery, obstetrics and gynecology, and emergency } \\
\text { medicine. }\end{array}$} \\
\hline \multicolumn{3}{|c|}{$\begin{array}{l}\star \star \star \text { Others include pediatrics, anesthesiology, ophthalmology, otorhinolaryngology, dermatology, } \\
\text { plastic surgery, radiology and psychiatry. }\end{array}$} \\
\hline \multicolumn{3}{|c|}{$\begin{array}{l}{ }^{\dagger} \text { Evaluation category means the average score by three third year EM residents for three-item survey } \\
\text { regarding their clinical attitude, knowledge and skill for EM, and positive participation to EM practice } \\
\text { on a scale of one to five with } 5 \text { being best. } 1 \text { means average score was greater than or equal to } 1 \text { and } \\
\text { less than } 2.2 \text { means average score was greater than or equal to } 2 \text { and less than } 3 \text {. } 3 \text { means average } \\
\text { score was greater than or equal to } 3 \text { and less than } 5 \text {. } 4 \text { means average score was greater than or } \\
\text { equal to } 4 \text { and less than } 5.5 \text { means average score was } 5 \text {. }\end{array}$} \\
\hline
\end{tabular}

The simulated patient was the same in both the first and last weeks of the rotation, which thus reduced as much as possible the bias due to individual anatomical differences. A normal human model can provide good interpretation for normal anatomical landmarks, but this model does not have abnormal findings. ${ }^{9}$

A letter of information was provided to PGY1 residents, which included the elements of informed consent.

\section{Assessment}

An 18-item, hands-on performance test (total score 21) was developed to test medical students' skills on the FAST exam (Fig. 1). ${ }^{10}$ The items and content on the test had been used previously. ${ }^{10}$ The test was administered to PGY1 residents individually in the first and last weeks of the EM rotation under the supervision of the principal investigator. Participation by PGY1 residents was voluntary and did not impact their status within the program. After finishing two months of the rotation, each PGY1 resident was assessed by three third-year EM residents (PGY3) using a three-item survey regarding their clinical attitude, knowledge and skills for EM, and positive participation in EM practice on a scale of one to five, with 5 being the best. ${ }^{11,12}$ The survey results are presented in Tables 1 and 2 . 
Table 2

Test results and evaluation of the postgraduate year 1 residents.

\section{Resident's identified specialty of interest at the time of rotation}

Internal medicine Surgery Others

First week

$\begin{array}{lll}\text { Mean score }(95 \% \mathrm{Cl}) \quad 7.50(5.18-9.82) & 6.78(5.85-7.70) & 7.94(6.83-9.05)\end{array}$

Last week

Mean $(95 \% \mathrm{Cl}) \quad 17.63(16.54-18.71) \quad 14.89(12.57-17.21) \quad 15.59(14.44-16.74)$

Difference

$\begin{array}{lll}\text { Mean }(95 \% \mathrm{Cl}) & 10.13(7.74-12.51) & 8.11(5.61-10.62)\end{array}$

Evaluation*

Clinical attitude

$\begin{array}{llll}\text { Mean }(95 \% \mathrm{Cl}) & 4.29(3.80-4.78) & 4.12(3.76-4.61) & 3.75(3.21-4.28)\end{array}$

Knowledge and skill

$\begin{array}{llll}\text { Mean }(95 \% \mathrm{Cl}) & 3.96(3.42-4.49) & 3.59(3.09-4.10) & 3.20(2.81-3.58)\end{array}$

Positive participation

$\begin{array}{llll}\text { Mean }(95 \% \mathrm{Cl}) & 4.13(3.60-4.65) & 3.81(3.11-4.52) & 3.49(3.00-3.98)\end{array}$

Total

$\begin{array}{llll}\text { Mean }(95 \% \mathrm{Cl}) & 4.13(3.64-4.61) & 3.86(3.37-4.36) & 3.48(3.04-3.92)\end{array}$

*Evaluation means that each PGY1 was assessed by three third year EM residents (PGY3) for threeitem survey regarding their clinical attitude, knowledge and skill for EM, and positive participation to EM practice on a scale of one to five with 5 being best.

\section{Statistical Analysis}

The primary outcome was the mean difference between the hands-on FAST examination scores of the first week of the EM rotation and the same hands-on FAST examination scores of the last week of the EM rotation. The subgroup analysis included factors affecting the change in the score using simple and multiple linear regression analyses. The same instructor and simulated patient were used for both handson performance tests of the study.

Values are reported as means and standard deviation for continuous variables, and as frequencies with percentages for categorical variables. Fisher's exact test was used for categorical variables. For the 
primary analysis, a paired t-test was used to examine differences in the hands-on FAST examination scores between the first week of the rotation and the last week of the rotation. A subgroup analysis according to the three categories of the identified specialty of interest at the time of the EM rotation of the PGY1 residents was performed, and the differences in the hands-on FAST examination scores were compared among the identified specialties of interest with multiplicity adjustment using Dunnett's method. The relative effects of baseline characteristics (subphrenic space identified on the left side, correct probe orientation on the right side, and the identified specialty of interest) on differences in the hands-on FAST examination scores over the 2 months were examined using a simple and multiple linear regression models.

All tests were 2-tailed, with $p<0.05$ considered significant. All analyses were performed with STATA (version 16.1; Stata Corp., College Station, TX, USA).

\section{Results}

A total of 34 PGY1 residents (male 29, female 5) were recruited and completed the hands-on FAST test in both the first and last weeks of the rotation (Table 1). None was lost to follow-up. There were no significant differences in residents' identified specialties by sex, group, and evaluation $(P=0.395, P=$ $0.169, \mathrm{P}=0.799$, respectively, Fisher's exact test; Table 1 ).

The mean test score for the PGY1 residents in the first week was 7.53 (standard deviation [SD] 2.12). The mean test score in the last week was 15.88 (SD 2.46). The primary outcome of this study was the score difference between the first and last weeks (mean 8.35, 95\% confidence interval [Cl] 7.36-9.35, P< 0.0001 , paired $t$-test).

The subgroup analysis by the identified specialty of interest at the time of the EM rotation showed that the FAST test score of the internal medicine group in the last week was superior to that of the surgery group ( $p=0.038$ after multiplicity adjustment), but there was no significant difference between the internal medicine group and the others group (Table 2, Fig. 2). In addition, there was no significant difference among the three groups in the FAST test of the last week.

Simple linear regression analysis showed that two items of the first week 'Correct probe orientation (indicator towards head) on the right side' and 'Correct probe orientation (indicator towards head) on the left side' were inversely correlated with the primary outcome (beta coefficient $(\mathrm{BC})=-3.03,95 \% \mathrm{Cl}-4.77-$ -1.29 and $\mathrm{BC}=-2.06,95 \% \mathrm{Cl}-3.97--0.14$, respectively); however, the same items in the last week were not predictive. Simple linear regression analysis also showed that two items in the last week 'Splenorenal recess identified' and 'Subphrenic space identified' were positive predictive factors for the primary outcome $(\mathrm{BC}=3.89,95 \% \mathrm{Cl} 1.97-5.82$ and $\mathrm{BC}=3.08,95 \% \mathrm{Cl} 0.97-5.19$, respectively), but the same items in the first week were not predictive. The results of multiple linear regression analysis are provided in Table 3. 'Correct probe orientation (indicator towards head) on the right side' in the first week, 'Subphrenic space identification' in the last week, and the identified specialty of interest at the time of the EM rotation were good prognostic factors for the primary outcome. 
Table 3

The result of multiple linear regression analysis.

\begin{tabular}{|c|c|c|c|c|c|}
\hline \multirow[b]{2}{*}{ Subphrenic space identified of left side } & \multirow{2}{*}{$\begin{array}{l}\begin{array}{l}\text { Beta } \\
\text { Coefficients }\end{array} \\
2.48\end{array}$} & \multirow{2}{*}{$\begin{array}{l}P \\
\text { value } \\
0.004\end{array}$} & \multicolumn{3}{|c|}{$\begin{array}{l}95 \% \text { Confidence } \\
\text { Interval }\end{array}$} \\
\hline & & & 0.85 & - & 4.12 \\
\hline $\begin{array}{l}\text { Correct probe orientation (indicator towards head) } \\
\text { of right side }\end{array}$ & -3.08 & $<.001$ & -4.48 & - & -1.68 \\
\hline \multicolumn{6}{|l|}{ Major } \\
\hline Internal medicine group & reference & & & & \\
\hline Surgery group & -1.67 & 0.092 & -3.63 & - & 0.29 \\
\hline Others group & -2.66 & 0.004 & -4.38 & - & -0.93 \\
\hline
\end{tabular}

\section{Discussion}

Hands-on practical FAST training for PGY1 residents improved their FAST test scores significantly during their 2-month-long EM rotation. The identified specialty of interest at the time of the rotation by the PGY1 residents seemed to affect the FAST test score. The PGY1 residents who chose internal medicine as their specialty tended to have better scores than the other two specialty groups, although the difference was not significant. There were several possible reasons why the internal medicine group was better than the other two groups on the FAST test. First, residents in the internal medicine group tended to participate in EM practice more than the other groups, so that they had more opportunities to perform FAST than the other two groups. In fact, the third-year EM residents found that PGY1 residents of the internal medicine group attended EM practice more than PGY1 residents of the other two groups (Table 2). Second, PGY1 residents of the internal medicine group were thought to practice much more than the other two groups. Third, some PGY1 residents of the internal medicine group selected gastroenterology as their specialty, and they might have learned abdominal ultrasound examination before their EM rotation. It was not possible to determine the reason why PGY1 residents of the surgery group were not superior to the other two groups, even though they were expected to learn FAST. ${ }^{13}$ One of the reasons might be that our hospital accepted a small number of trauma patients.

Simple linear regression analysis showed that two items (correct probe orientation (indicator towards head) on the right and left sides) in the first week were inversely correlated with the primary outcome. PGY1 residents who could perform correct probe orientation (indicator towards the head) bilaterally tended to show high scores in the first week, and, thus, they could not improve their score in the last week. For the same reason, two items (splenorenal recess identification and subphrenic space identification) in the last week were difficult to perform, and these positions were the key for a high score. Thus, some PGY1 residents who could not perform correct probe positioning in the first week could eventually 
perform both splenorenal recess identification and subphrenic space identification showed higher scores. Multiple linear regression analysis showed that PGY1 residents who failed 'Correct probe orientation (indicator towards head) on the right side' in the first week, who could perform 'Subphrenic space identification' in the last week and had selected internal medicine as their future specialty tended to have a higher primary outcome (Fig. 2).

This study has several limitations. First, the identified specialty of interest at the time of EM rotation of the PGY1 residents was divided arbitrarily into 3 groups. Surgical specialties in our category have to see trauma patients in the emergency room, whereas internal medicine and others do not have to see trauma patients, and internal medicine physicians have to use ultrasound basically in their practice; thus, we believe these classifications were reasonable. Second, the number of PGY1 residents was small, especially the number of female residents, which was only five; thus, sex was not considered as a predictor of FAST performance. Third, all PGY1 residents were told they would take the second test in the last week of their rotation; thus, some of them practiced FAST just before the test, which might have affected their scores. Actually, some PGY residents whose second test result was higher than average failed to perform FAST adequately for real trauma patients after their EM rotation; therefore, PGY1 residents should have been evaluated one more time after the EM rotation.

In conclusion, practical FAST training for PGY1 residents during the EM rotation significantly improved their FAST test scores. Although it was not significant, the identified specialty of interest of the PGY1 residents at the time of the EM rotation seemed to affect the FAST test score.

\section{Declarations}

\section{Acknowledgements:}

not applicable

\section{Contributions:}

$\mathrm{KOb}$ and $\mathrm{YI}$ treated the patient and made the clinical diagnosis, $\mathrm{KoO}, \mathrm{KaO}$ and $\mathrm{AT}$ wrote and revised the manuscript. All authors have read and approved the manuscript.

\section{Funding:}

This research did not receive any specific grant from funding agencies in the public, commercial, or notfor-profit sectors.

\section{Availability of data and materials:}


The datasets used and/or analysed during the current study available from the corresponding author on reasonable request.

\section{Conflict of interest:}

The authors declare that they have no competing interests.

\section{Informed consent:}

We obtained verbal and written informed consent from the patient for reporting this case.

\section{Ethical approval and Human rights statement:}

The research was approved and consent to participate was obtained by the Institutional Review Board at Osaka Medical College. (number 2767).

\section{References}

1. Ma OJ, Gaddis G, Norvell JG, Subramanian S. How fast is the focused assessment with sonography for trauma examination learning curve? EMA - Emerg Med Australas. 2008;20(1):32-37. doi:10.1111/j.1742-6723.2007.01039.x

2. Rose JS. Ultrasound in abdominal trauma. Emerg Med Clin North Am. 2004;22(3):581-599. doi:10.1016/j.emc.2004.04.007

3. Savatmongkorngul S, Wongwaisayawan S, Kaewlai R. Focused assessment with sonography for trauma: current perspectives. Open Access Emerg Med. 2017;9:57-62. doi:10.2147/OAEM.S120145

4. Gogalniceanu P, Sheena Y, Kashef E, Purkayastha S, Darzi A, Paraskeva P. Is basic emergency ultrasound training feasible as part of standard undergraduate medical education? J Surg Educ. 2010;67(3):152-156. doi:10.1016/j.jsurg.2010.02.008

5. Onishi H. History of Japanese medical education The introduction of Western-style med-ical education. Korean J Med Educ. 2018;30(4):283-294. doi:10.3946/kjme.2018.103

6. Favot M, Courage $\mathrm{C}$, Mantouffel J, Amponsah D. Ultrasound training in the emergency medicine clerkship. West J Emerg Med. 2015;16(6):938-942. doi:10.5811/westjem.2015.9.27290

7. Stolz LA, Stolz U, Fields JM, et al. Emergency Medicine Resident Assessment of the Emergency Ultrasound Milestones and Current Training Recommendations. Acad Emerg Med. 2017;24(3):353361. doi:10.1111/acem.13113

8. Østergaard ML, Rue Nielsen K, Albrecht-Beste E, Kjær Ersbøll A, Konge L, Bachmann Nielsen M. Simulator training improves ultrasound scanning performance on patients: a randomized controlled trial. Eur Radiol. 2019;29(6):3210-3218. doi:10.1007/s00330-018-5923-z 
9. Mohammad A, Hefny AF, Abu-Zidan FM. Focused Assessment Sonography for Trauma (FAST) training: a systematic review. World J Surg. 2014;38(5):1009-1018. doi:10.1007/s00268-013-2408-8

10. Poland S, Frey JA, Khobrani A, et al. Telepresent focused assessment with sonography for trauma examination training versus traditional training for medical students: A simulation-based pilot study. J Ultrasound Med. 2018;37(8):1985-1992. doi:10.1002/jum.14551

11. Martin J, Lloyd M, Singh S. Professional attitudes: can they be taught and assessed in medical education? Clin Med. 2003;2(3):217-223. doi:10.7861/clinmedicine.2-3-217

12. Jauregui J, Gatewood MO, Ilgen JS, Schaninger C, Strote J. Emergency medicine resident perceptions of medical professionalism. West J Emerg Med. 2016;17(3):355-361. doi:10.5811/westjem.2016.2.29102

13. Beal EW, Sigmond BR, Sage-Silski L, Lahey S, Nguyen V, Bahner DP. Point-of-Care Ultrasound in General Surgery Residency Training: A Proposal for Milestones in Graduate Medical Education Ultrasound: A. J Ultrasound Med. 2017;36(12):2577-2584. doi:10.1002/jum.14298

\section{Figures}




\section{Focused Assessment with Sonography for Trauma score sheet}

Yes (1) No (0)

\section{Peristernal Cardiac ( 4 points)}

Correct probe orientation(indicator to right shoulder)

Image anatomy

Heart Identified

Pericardial sac identified

Aorta identified

Right Upper Quadrant (5 points)

Correct probe orientation(indicator towards head)

Image anatomy

Diaphram identified

Morrison's Pouch identified

Tip of Liver identified

Inferior pole of right kidney identified

$\begin{array}{ll}1 & 0 \\ 1 & 0 \\ 1 & 0 \\ 1 & 0\end{array}$

Left Upper Quadrant (5 points)

Correct probe orientation(indicator towards head)

1

Image anatomy

Diaphram identified

Subphrenic space identified

Splenorenal recess identified

Inferior pole of right kidney identified

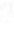

Pelvic (4 points)

Correct probe orientation for transverse(indicator to right)

Correct probe orientation for sagittal(indicator towards head)

Image anatomy

Bladder identified

Pouch of Douglas identified (Rectouterine, Retrovescicular)

0

$$
0
$$



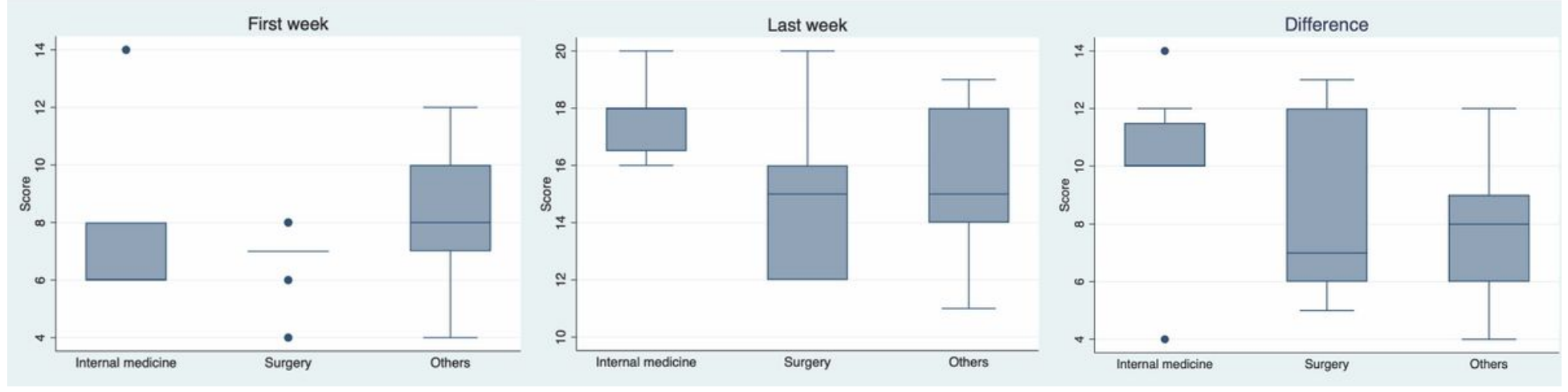

Figure 2

There was no significant difference between the internal medicine group and the others group. 